\title{
The Influence of Sub-Wavelength Effective Refractive Index Layer on the Transmittance of LYSO Scintillator ${ }^{\dagger}$
}

\author{
P. Modrzyński ${ }^{a, *}$, A. OlejniczAK ${ }^{a}$, A. ZięBA ${ }^{a}$, P. Kunicki $^{a}$, M. TOMAniK $^{b}$ \\ AND M. WIELEBSKI ${ }^{a}$
}

${ }^{a}$ Nanores Sp. z o.o. Sp. k., Bierutowska 57-59, 53-317 Wrocław, Poland

${ }^{b}$ Department of Biomedical Engineering, Mechatronics and Theory of Mechanisms, Faculty of Mechanical Engineering, Wrocław University of Science and Technology, Łukasiewicza 7/9, 50-371 Wrocław, Poland

(Received September 11, 2016)

From various types of scintillating materials lutetium-yttrium oxyorthosilicate (LYSO) has the highest luminosity and the greatest potential of application in high-energy radiation detectors. Due to the small critical angle of total internal reflection the enhancement of the extraction of light outside a scintillator is a challenge. We study numerically the influence of the effective refractive index layer on the transmittance of LYSO crystal. It is possible to realize such layer by sub-wavelength patterning of crystal surface using for example focused ion beam. The enhancement of transmittance of LYSO crystal up to $100 \%$ as well as the possibility to tune the positions of transmittance maxima have been shown.

DOI: 10.12693/APhysPolA.131.1565

PACS/topics: 42.25.Bs, 42.25.Gy, 42.79.Dj, 02.70.Bf, 78.70.Ps, 29.40.Mc

\section{Introduction}

Scintillators are materials with the capability to absorb ionizing radiation, such as $\alpha$-, $\beta$ - particles or $\gamma$ - and $\mathrm{X}$-ray, and to convert a part of the absorbed energy into visible or ultraviolet light. The basic process is called scintillation, it comes from the Latin word "scintillare" which means sparkle or twinkle [1]. The conversion process usually takes from nano- to microseconds and produce short pulse of photons corresponding to proper ionizing radiation. Generally the intensity of the light pulse is proportional to the energy absorbed by the scintillator, and could be sensed by detector and converted into a physical signal for example electrical [2]. Scintillator may be solid or liquid, crystalline or non-crystalline and organic or inorganic. Different kind of the scintillator are used in various types of detectors. Organic plastic and liquid scintillators are used to detect high-energy neutrons in the presence of gamma radiation background $[3,4]$. The inorganic crystal scintillators are ideal for detection of $\mathrm{X}$ - and $\gamma$-ray, their higher density and atomic number $Z$ cause better detection efficiency. One may find a number of patents for scintillating radiation detectors $[5,6]$, which are used for example in medical application such as computer tomography, radiology, positron emission tomography (PET) and many others [7].

Due to their good detection efficiency, inorganic crystal scintillators are the most popular. In the past years enormous effort was made to develop more efficient scin-

*corresponding author; e-mail: pawel.modrzynski@nanores.pl

${ }^{\dagger}$ paper contributed to the 11th Polish-Japanese Joint Seminar on Micro and Nano Analysis, Gniew, Poland, September 11-14, 2016 tillators for ion radiation detection, for example BGO, GSO, or LYSO [2].

TABLE I

Physical and optical properties of chosen scintillators $[2,8]$.

\begin{tabular}{c|c|c|c|c}
\hline \hline Parameter & Unit & LYSO & BGO & GSO \\
\hline melting point & {$\left[{ }^{\circ} \mathrm{C}\right]$} & 2050 & 1050 & 1900 \\
density & {$\left[\frac{\mathrm{g}}{\mathrm{cm}^{3}}\right]$} & 7.11 & 7.13 & 6.71 \\
effective $Z$ & - & 64 & 73 & 60 \\
refractive index & - & 1.82 & 2.15 & 1.87 \\
peak emission & {$[\mathrm{nm}]$} & 420 & 480 & 430 \\
decay constant & {$[\mathrm{ns}]$} & $40-44$ & 300 & 50 \\
radiation length & {$[\mathrm{cm}]$} & 1.10 & 1.1 & 1.38 \\
critical angle & {$\left[{ }^{\circ}\right]$} & 33.33 & 27.72 & 32.33 \\
luminosity & {$\left[\frac{\text { photons }}{\text { MeV }}\right]$} & 34.000 & 8.200 & 7.400
\end{tabular}

In this paper we are interested in one of the Ce-doped silicates, LYSO. This material compared to other popular scintillators has desirable optical and physical properties such as luminosity, see Table I [8].

On the other hand, while the optoelectronic sensor collects signal in a small range, scintillator materials are commonly isotropic, this means that generated visible light is emitted at all possible angles. The light transmission is limited by the Fresnel reflection due to different refractive index between two materials [9]. Additionally, the extraction efficiency may be limited in other ways, the produced light inside the material is trapped inside the crystal due to total internal reflection (TIR). According to Snell's law, light can only pass into a medium with a lower index of refraction (ambient) when the incident angle $\theta$ is smaller than the critical angle $\theta_{c}[10]$. Extraction of light to the outside of scintillator crystal is an issue. 
Recently a great potential of the photonic crystals $(\mathrm{PhC})$ has been proven, in order to increase the efficiency of scintillator-based detectors, that $\mathrm{PhC}$ is an optical nanostructure which periodically changes the refractive index in one, two, or three dimensions. The extraction of light outside the crystal is influenced by the periodic arrangement of the $\mathrm{PhC}$. The light is scattered at the interfaces where the refraction index changes, the scattered light waves are able to interfere with each other (destructively or constructively). One may find number of theoretical and experimental papers devoted to photonic crystals patterns on the surface of LYSO crystals to increase the efficiency of scintillator detectors [10-13].

Sub-wavelength gratings (SWG) [14] also may be used to change the crystal optical properties. Since these biomimetic structures were found first at the eyes of moths [15] the SWG were studied both experimentally and numerically as color filters [16, 17] or antireflectance $[18,19]$ structures. SWG are periodic structures with period smaller than wavelength of incident light, therefore they show emergent optical properties [14]. For example light going through SWG with wavelength larger than period behaves like passing through a layer with effective refractive index [20]. There are several different models of calculation of the effective properties of subwavelength structures. Effective optical properties are strongly dependent on the shape of SWG [20], as is shown in Fig. 1.

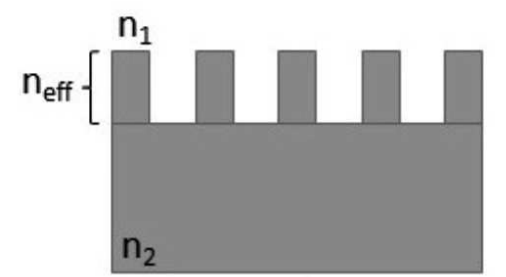

rectangular SWG

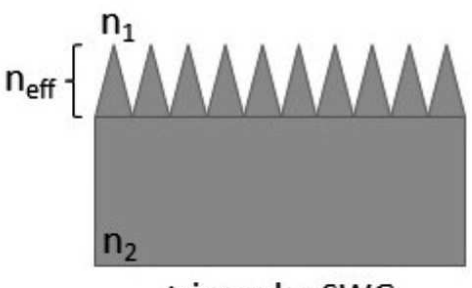

triangular SWG
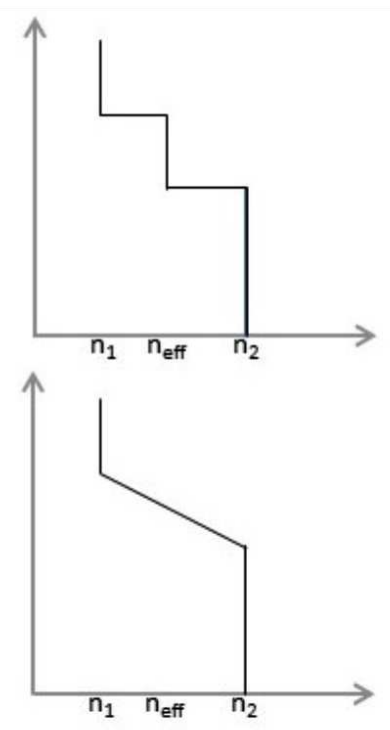

Fig. 1. Different possibilities of SWG structures and their effective refractive index profiles.

For rectangular-type SWG (Fig. 1) the simple linear criterion [21] of calculation of effective refractive index can be adapted

$$
n_{\text {eff }}=(1-\phi) n_{\text {air }}+\phi n_{\text {crystal }},
$$

where $\phi$ is a parameter from the range $(0,1)$ and indicates the part of a period occupied by a crystal.
The aim of the study was to conduct numerical simulation of the SWG patterned LYSO crystals in order to determine the light extraction improvement.

\section{Calculations details}

Analysis of the electromagnetic wave leaving LYSO crystal into air was studied numerically using commercial OptiFDTD package (freeware 32-bit version available from OptiWave website) within finite difference time domain (FDTD) method. Our calculations were divided into two parts. Firstly we considered the layer with effective refractive index $n_{\text {eff }}$ from the range $\left(n_{\text {air }}, n_{\mathrm{LYSO}}\right)$ and its influence on the transmittance of the LYSO crystal surface. Secondly we considered the one-dimensional rectangular pattern with sub-wavelength (SW) period as a possible realization of SWG with effective refractive index. Simulated layout is presented in Fig. 2a for effective refractive index layer and in Fig. $2 \mathrm{~b}$ for $\mathrm{SW}$ patterned crystal periodic boundary conditions (PBC) were applied to simulate infinite surface of LYSO crystal and anisotropic perfectly matched layers (APML) were applied in direction of the wave propagation to ensure the absorption of the electromagnetic wave leaving the simulation wafer. The wave coming out from LYSO crystal was a Gaussian modulated continuous wave (GMCW) centered at $400 \mathrm{~nm}$ with half width equal to $7.5008 \times 10^{-16} \mathrm{~s}$ and time offset equal to $2.6495 \times 10^{-15} \mathrm{~s}$ and its origin was placed $1 \mu \mathrm{m}$ below the crystal surface (red line in Fig. 2a,b). We considered only TE mode of electromagnetic wave incident normally at the boundary between LYSO crystal and additional (effective or sub-wavelength) layer. Transmitted power was collected by observation line (green line in Fig. 2) placed $1 \mu \mathrm{m}$ above the layer and it was normalized with the input power of electromagnetic wave. We used fixed values of refractive indices for LYSO crystal as well as for effective layer. No spectral dependence was considered. Refractive index for LYSO crystal was 1.82. Effective refractive indices for the layer were chosen from the range between air and LYSO crystal (1.001.82 ) with a $10 \%$ step in the first part of the calculations. Then the effective refractive index of the layer was fixed at value 1.328 (corresponding to the SWG layer containing $40 \%$ of LYSO crystal) and layer thickness $(H)$ was changed in range of $0.1 \mu \mathrm{m}$ to $1.0 \mu \mathrm{m}$ with $0.1 \mu \mathrm{m}$ step. In the second part of the calculations we considered the periodic rectangular structuration with $\mathrm{SW}$ periods $P=\left(0.1 \lambda_{\mathrm{LYSO}}, 0.2 \lambda_{\mathrm{LYSO}}, 0.3 \lambda_{\mathrm{LYSO}}\right)$, where $\lambda_{\mathrm{LYSO}}$ indicates the peak emission wavelength of the LYSO crystal being equal to $420 \mathrm{~nm}$. Width of the pattern $(w)$ was set to $0.4 P$. Thickness of SW pattern $(H)$ was changed from $0.1 \mu \mathrm{m}$ to $1.0 \mu \mathrm{m}$ with $0.02 \mu \mathrm{m}$ step. For all calculations the mesh unit was set to $0.01 \mu \mathrm{m}$ but for the calculation of the pattern with $P=0.1 \lambda_{\text {LYSO }}$ the mesh unit was decreased to $0.0025 \mu \mathrm{m}$. Total simulation time was 3000 steps or 6000 steps for the calculations of effective layers or patterns, respectively. Time step was equal to $2.22376 \times 10^{-17} \mathrm{~s}$. 
(a)

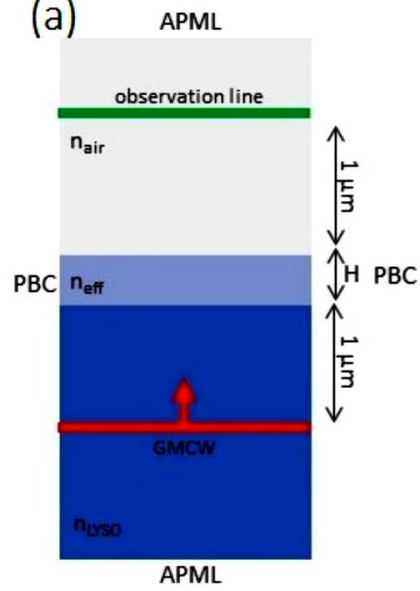

Fig. 2. Simulation layout of (a) LYSO crystal with effective refractive index layer and (b) LYSO crystal with one-dimensional rectangular sub-wavelength structuration. See text for detailed explanation of symbols.

\section{Results and discussion}

At first we have considered $0.2 \mu \mathrm{m}$ thick layer with effective refractive index from the range between 1.00 (air) and 1.82 (LYSO) with $10 \%$ step. Reduction of the refractive index by $10 \%$ can be interpret as making a pattern with $10 \%$ less amount of LYSO crystal in a period, for simple patterns (e.g. rectangular SWG, Fig. 1). The results are shown in Fig. 3. It can be seen that comparing to the transmittance of pure LYSO crystal, which is about $91 \%$ at normal incidence, sub-wavelength structuring of a sample can enhance the transmission of light outside LYSO crystal up to about $100 \%$. Transmittance of LYSO+effective layer system is wavelength-dependent showing both regions of great enhancement as well as minima, where transmittance equal to the transmittance of pure LYSO crystal.

Layer with effective refractive index equal to 1.738 (corresponding to the lack of $10 \%$ of LYSO crystal in a period) results in small transmittance enhancement (about 2\% higher at the maximum). Further decrease of effective refractive index (corresponding to further removal of LYSO material from the SWG period) results in further enhancement of transmittance. In our case the layer with effective refractive index equal to 1.328 shows the highest transmittance maximum being almost $100 \%$. Further decrease of effective refractive index results also in smaller maximum transmittance.

Then we performed calculations considering effective layer with highest transmittance. Effective refractive index was fixed at 1.328 and the thickness of the layer (or the depth of the structuration) was changed from $0.1 \mu \mathrm{m}$ up to $1.0 \mu \mathrm{m}$ with $0.1 \mu \mathrm{m}$ step. Observation line was placed $1 \mathrm{\mu m}$ above the layer for all thicknesses. The results are gathered all together in Fig. 4 and connected in pairs on every sub-plot to better present the transmittance change with increase of the thickness. It can

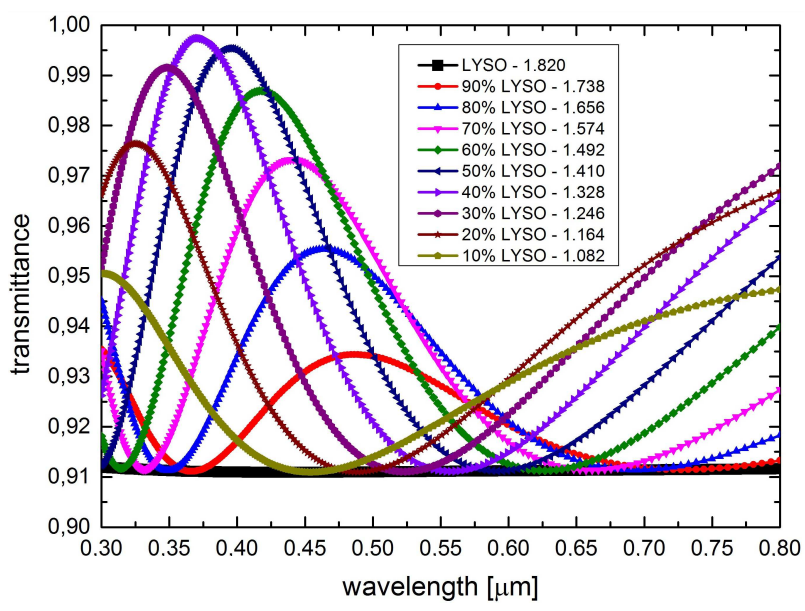

Fig. 3. Transmittance spectra for layers with different effective refractive indices compared with pure LYSO crystal.

be noticed that the transmittance spectrum has strongly oscillatory character showing peaks with maxima about $100 \%$ and minima equal to the transmittance of pure LYSO crystal. Also the peaks are getting narrower in the short waves region of considered spectrum. It can be also observed that when the layers differ by $0.1 \mu \mathrm{m}$ their transmittance spectra are almost opposite i.e. the maximum of transmittance of thinner layer is placed almost at the minimum of the thicker layer. For very thick layers (above $0.8 \mu \mathrm{m}$ ) overlapping of peaks in UV region for two different layers can be seen. For effective layer with thickness equal to $0.7 \mu \mathrm{m}$ a transmittance peak centered at $420 \mathrm{~nm}$ was obtained which is a wavelength emitted by LYSO crystal after high energy radiation excitation.

In the second part we calculated the transmittance spectra for the LYSO crystal surface with periodic, onedimensional SW structuration of rectangular type, as shown in Fig. 2b. The LYSO crystal emission peak wavelength $\lambda_{\text {LYSO }}=420 \mathrm{~nm}$ has been chosen as our wavelength of interest for which the patterned surface should reveal the highest transmittance. Width of the rectangular pattern $(w)$ was set to $40 \%$ of period, therefore proposed SW pattern corresponds to the effective layer with $n_{\text {eff }}=1.328$ and the thickness $(H)$ was fixed as $0.2 \mu \mathrm{m}$. Three different periods equal to $0.1 \lambda_{\mathrm{LYSO}}, 0.2 \lambda_{\mathrm{LYSO}}$, and $0.3 \lambda_{\text {LYSO }}$, were investigated. We decided to not enlarge the periods to maintain the sub-wavelength character of the pattern. Figure 5 presents obtained transmittance spectra compared with the spectrum for effective layer with $n_{e f f}=1.328$. Good agreement of all spectra is clearly seen, and the spectrum for period $0.2 \lambda_{\mathrm{LYSO}}$ reveals the best fit to the effective layer in the shortwavelength regime. We found that patterns with wider periods $\left(0.4 \lambda_{\text {LYSO }}\right.$ and wider $)$ show more deviations from effective layer spectrum and decrease in transmittance. When the length of the period is comparable to the wavelength, diffraction phenomenon occurs resulting in the transmittance losses. 


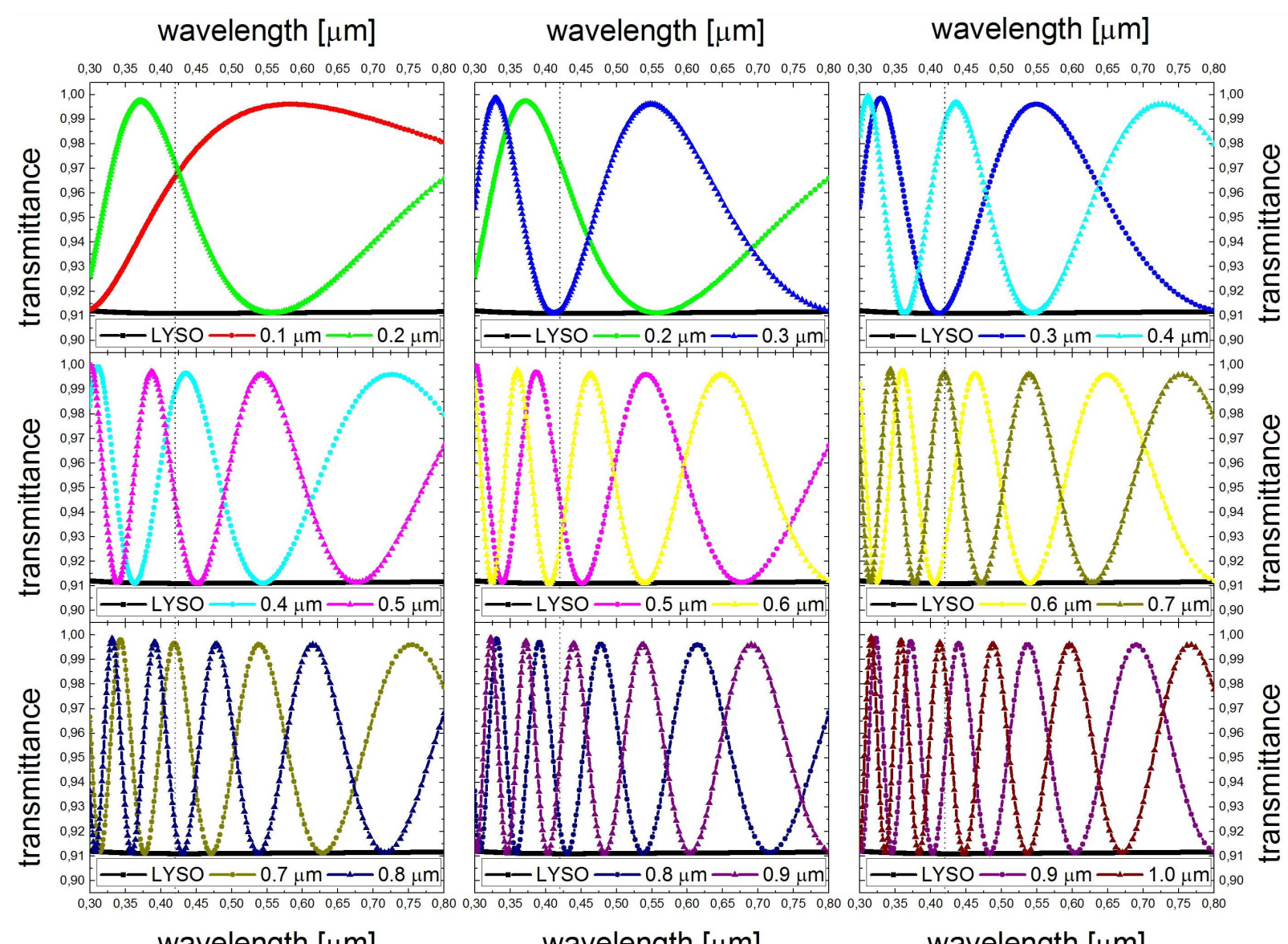

wavelength $[\mu \mathrm{m}]$

wavelength $[\mu \mathrm{m}]$

wavelength $[\mu \mathrm{m}]$

Fig. 4. Transmittance spectra for layers with fixed effective refractive index equal to 1.328 and for different thicknesses. Spectra are gathered in pairs to better see the change in transmittance with increase of the thickness by $0.1 \mu \mathrm{m}$. Transmittance spectra for the same layer thickness are given in the same color. Dashed line at $0.42 \mu \mathrm{m}$ is the emission line of LYSO crystal.

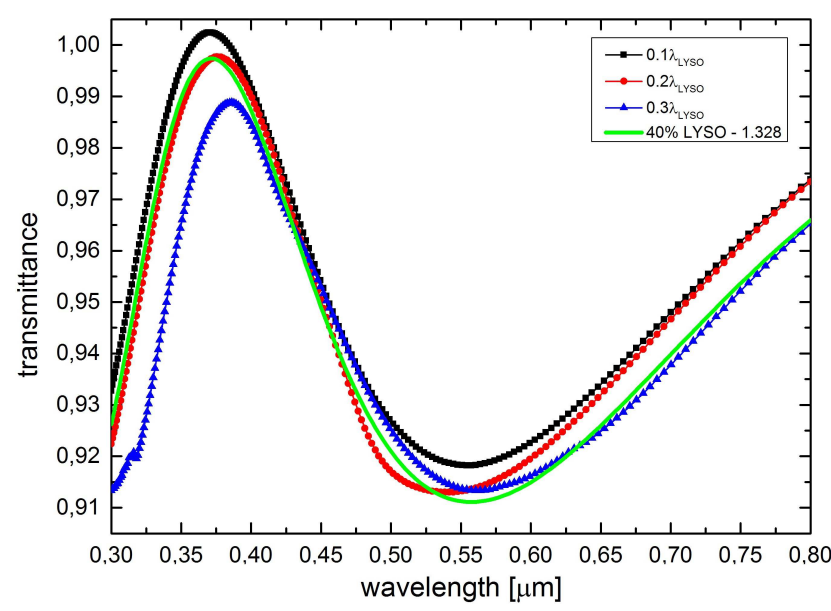

Fig. 5. Transmittance spectra for LYSO crystal with sub-wavelength rectangular structuration with periods equal to $0.1 \lambda_{\text {LYSO }}$ (black points), $0.2 \lambda_{\text {LYSO }}$ (red points), and $0.3 \lambda_{\text {LYSO }}$ (blue points), where $\lambda_{\text {LYSO }}=420 \mathrm{~nm}$. Transmittance spectrum for layer with effective refractive index equal to 1.328 is shown for comparison.

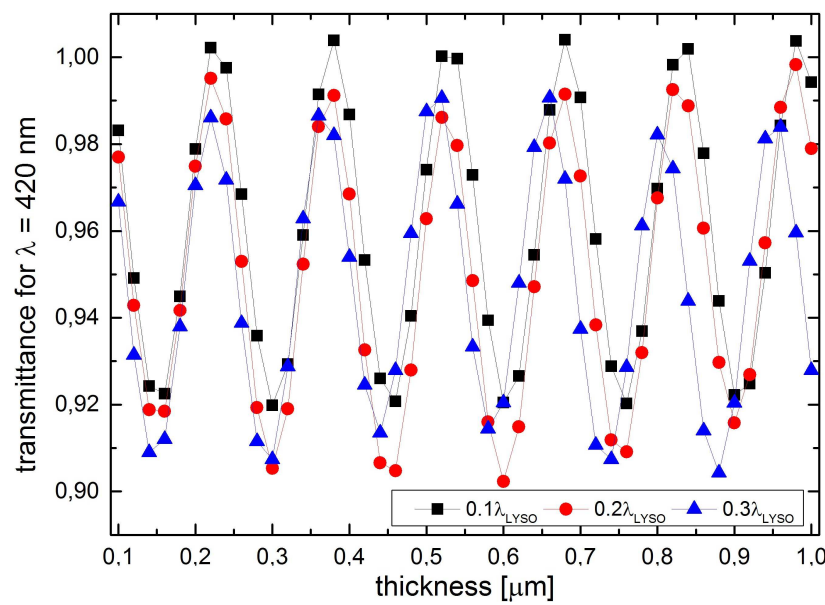

Fig. 6. Transmittance of the LYSO crystal with subwavelength structuration with different periods and thickness for $\lambda=420 \mathrm{~nm}$.

From the previous part of our calculations we found that the spectral position of the transmittance maxima can be tuned with the change of the effective layer 
thickness. Therefore we repeated the transmittance calculation for different thicknesses of SW pattern from the range of $0.1 \mu \mathrm{m}$ to $1.0 \mu \mathrm{m}$ with $0.02 \mu \mathrm{m}$ step. The transmittance for the $\lambda_{\mathrm{LYSO}}=420 \mathrm{~nm}$ for different period and thicknesses are shown in Fig. 6. It can be seen that almost $100 \%$ transmission for $\lambda_{\mathrm{LYSO}}=420 \mathrm{~nm}$ can be obtained for patterns with thicknesses $0.22,0.38,0.52$, $0.68,0.82$, and $0.98 \mu \mathrm{m}$. The position of transmission maxima for $\lambda_{\mathrm{LYSO}}$ are equal for all considered periods.

\section{Conclusions}

In this work the calculations of transmittance spectra of LYSO crystal with additional effective layer for normal incidence within FDTD method were presented. After that, the sub-wavelength rectangular pattern was proposed as a possible realization of the layer with effective refractive index. We showed that the sub-wavelength structuration of crystal surface can enhance the transmittance up to almost $100 \%$ for the layer with effective refractive index equal to 1.328 corresponding to the rectangular sub-wavelength pattern with $40 \%$ of LYSO crystal. We found that the spectral position of transmittance peaks can be precisely tuned by changing the thickness of the SWG layer.

\section{Acknowledgments}

The work was carried out by Nanores Numerical Simulations Division.

\section{References}

[1] A. Knapitsch, Ph.D. Thesis, Vienna University of Technology, Vienna 2012.

[2] C.L. Melcher, J. Nucl. Med. 41, 1051 (2000).

[3] N. Zaitseva, B.L. Rupert, I. Pawełczak, A. Glenn, H.P. Martinez, L. Carman, M. Faust, N. Cherepy, S. Payne, Nucl. Instrum. Methods Phys. Res. A 668, 88 (2012).
[4] D.L. Horrocks, Appl. Spectrosc. 24, 397 (1970).

[5] D.F. DeCou, T. Roach, United States Patent No. US 4,234,792 (1980).

[6] M.M.C. Chou, United States Patent No. US 8,158,948 B2 (2012).

[7] S.J. Duclos, Electrochem. Soc. Interface 7, 34 (1998).

[8] L. Pidol, A. Kahn-Harari, B. Viana, E. Virey, B. Ferrand, P. Dorenbos, J.T.M. De Haas, C.W.E. Van Eijk, IEEE Trans. Nucl. Sci. 51, 1084 (2004).

[9] J.-G. Kim, C.-H. Hsieh, H.J. Choi, J. Gardener, B. Singh, A. Knapitsch, P. Lecoq, G. Barbastathis, Opt. Express 23, 22730 (2015).

[10] A. Knapitsch, E. Auffray, C.W. Fabjan, J.L. Leclercq, P. Lecoq, X. Letartre, C. Seassal, Nucl. Instrum. Methods Phys. Res. A 628, 385 (2011).

[11] Z. Wang, Y. Hao, Z. Wang, X. Liu, Q. Zhang, D. Zhu, Sci. World J. 2012, 515468 (2012).

[12] A. Knapitsch, P. Lecoq, Int. J. Mod. Phys. A 29, 1430070 (2014).

[13] P. Modrzynski, T. Gotszalk, A. Knapitsch, P. Kunicki, P. Lecoq, M. Moczala, I. Papakonstantinou, E. Auffray, IEEE Trans. Nucl. Sci. 63, 644 (2016).

[14] R. Halir, P.J. Bock, P. Cheben, A. OrtegaMonux, C. Alonso-Ramos, J.H. Schmid, J. Lapointe, D.X. Xu, J.G. Wanguemert-Perez, I. MolinaFernandez, S. Janz, Laser Photon. Rev. 9, 25 (2015).

[15] A.R. Parker, H.E. Townley, Nat. Nanotechnol. 2, 347 (2007).

[16] B.H. Cheong, O.N. Prudnikov, E. Cho, H.S. Kim, J. Yu, Y.S. Cho, H.Y. Choi, S.T. Shin, Appl. Phys. Lett. 94, 213104 (2009).

[17] Y.-T. Yoon, H.-S. Lee, S.-S. Lee, S.H. Kim, J.D. Park, K.-D. Lee, Opt. Expr. 16, 2374 (2008).

[18] K. Han, C.-H. Chang, Nanomaterials 4, 87 (2014).

[19] N. Das, S. Islam, Energies 9, 690 (2016).

[20] S. Chattopadhyay, Y.F. Huang, Y.J. Jen, A. Ganguly, K.H. Chen, L.C. Chen, Mater. Sci. Eng. R Rep. 69, 1 (2010).

[21] M.M. Braun, L. Pilon, Thin Solid Films 496, 505 (2006). 